\title{
Chronogeometrical Determinism and the Local Present
}

\author{
Steven F. Savitt*
}

1. The argument for chronogeometrical determinism.

In time-oriented Minkowski spacetime let us choose some point or event and call it $\mathrm{O}$. Then for any other point or event $\mathrm{e}$ in such a spacetime either (a) e is itself spacelike separated from $\mathrm{O}$ or (b) there is some third point $\mathrm{e}^{\prime}$ which is spacelike separated from both $\mathrm{O}$ and e. In case (a) there is some inertial coordinate system in which $\mathrm{O}$ and $\mathrm{e}$ are assigned the same time coordinate. Another common way of stating this fact, especially in popular books on the special theory of relativity, is that there is some inertial "observer" for whom $\mathrm{O}$ and e are simultaneous.

In case (b) there is some inertial coordinate system in which $\mathrm{O}$ and e' are assigned the same time coordinate and a second, different inertial coordinate system in which e' and e are assigned the same time coordinate (according to that second inertial coordinate system). A common alternative way of saying the same thing is that there is an inertial coordinate system $\mathrm{F}$ in which $\mathrm{O}$ and $\mathrm{e}^{\prime}$ are simultaneous and a second inertial coordinate system $F^{\prime}$ in which e' and e are simultaneous.

These are undisputed facts about (time-oriented) Minkowski spacetime, typically proven in rigorous texts, sometimes given as exercises. From time to time these facts have provoked curious reflections in the minds of reflective physicists and philosophers.

What are these curious reflections? If two events are simultaneous, they happen at the same time. If two events happen at the same time, it would seem that it must be the case that if one is actual, determinate, or real (whatever one means by these words), then the other must be actual, determinate, or real as well. Let me suppose that I am at presently located at $\mathrm{O}$. It is difficult to deny that I at present am actual, determinate, or

\footnotetext{
* Department of Philosophy, University of British Columbia, Vancouver, British Columbia, Canada, V6T 1Z1. I am greatly indebted to Richard Arthur and Dennis Dieks for their many helpful comments on a previous draft of this essay.
} 
real, and so it must be in case (a) above that that the simultaneous event e is actual, determinate, or real.

In the slightly more complex case (b), the reasoning of the previous paragraph from event $\mathrm{O}$ to event $\mathrm{e}^{\prime}$ leads me to the conclusion that $\mathrm{e}^{\prime}$ is actual, determinate, or real. But of course e' is simultaneous with e for a second inertial observer, and we are taught that in the special theory of relativity all inertial observers are to be treated equally. There are no privileged observers. So if we imagine the second observer passing through or experiencing event e', we can imagine that that second observer, whom we will call Bob, takes himself then (that is, at event e') to be actual, determinate, or real. Then of course e, being simultaneous with e', must also be actual, determinate, or real (for Bob). But Bob's view is as good as mine or yours or anyone's, since there are no privileged observers. We ought to be able to leave Bob out of the picture and just say that e is actual, determinate, or real, period.

But then it occurs to one that $\mathrm{O}$ and e were chosen arbitrarily. $\mathrm{O}$ can be any event in the spacetime, and e can be any other event, say an event far to the future of O. Have we not just shown then that insofar as we think of $\mathrm{O}$, the present instant, as actual, determinate, or real, the geometric features of Minkowski spacetime and the symmetries of the special theory or relativity force us to believe that any other event in such a spacetime, no matter how far to the future, is similarly actual, determinate, or real? All events, then, must be equally actual, determinate, or real. This view, held as a result of the argument sketched above, I call chronogeomtrical determinism. ${ }^{1}$

It would seem to follow that there is no coming into being or happening if chronogeometrical determinism is true - that is, if all events are equally actual,

\footnotetext{
${ }^{1}$ Determinism and fatalism are two variants of the view that the future is fixed and unalterable. For fatalism the fixity flows from logic and the correspondence theory of truth, whereas arguments for determinism involve laws of nature and the lawful evolution of states of systems. The view I have called chronogeometrical determinism falls awkwardly between these two types; but since it involves more than logic and correspondence, I think of it as more akin to classic determinism than prototypical fatalism. Nothing hangs on the terminology, but it is useful to be reminded that determinism and fatalism are distinct.
} 
determinate, or real. If there is no coming into being or happening, then there is no passing of time or temporal becoming. These latter notions, it would appear, are notions demonstrably inapplicable in Minkowski spacetime and must be dismissed as some sort of illusion or prejudice of untutored common sense.

While, as I said, this argument (or some variant of it) has occurred to several reflective individuals, ${ }^{2}$ it has occurred to other reflective individuals that this argument is flawed. ${ }^{3}$ Their criticisms fall into two broad categories. There are negative responses, arguments that try to show that at one point or another the arguments of RPPM are unsound, invalid, or somehow off the mark. There are also positive responses, attempts to provide an account of becoming in Minkowski spacetime that would, if successful, provide a forthright counterexample to the conclusions of RPPM. The aim of this chapter is to elaborate a response of the latter sort, but it will be worthwhile to take a brief look first at the negative responses. ${ }^{4}$

Prior to the advent of relativity, there were several ideas about time and reality that naturally fit together. If one thinks in terms of events, the existence or, if you like, the reality of an event is its happening. Two events that happen at the same time (as indicated, say, by synchronized clocks at the location of each event) are both (at that time) happening, are both real. Sameness of time, or simultaneity, is a global and nonrelative matter. These concepts naturally coalesce into a widely shared metaphysical view, the view of the "man in the street" according to Putnam $(1967,198)$, called presentism:

(P) All (and only) things that exist now are real.

\footnotetext{
${ }^{2}$ Rietdijk (1966. 1976), Putnam (1967), Penrose (1989, 2001), Maxwell (1985, 1988). I will refer to this group of authors as RPPM. There are many differences of detail and style between these authors, but I believe that the argument I present in the text captures a common or underlying core of their thinking. 
After the advent of relativity, these ideas concerning time and reality diverge. Sameness of time, simultaneity, becomes relative to choice of (amongst other things) a timelike line or time axis. Having chosen our event $\mathrm{O}$ in the initial argument, we are free to choose any one of a non-denumerable infinity of straight lines through $\mathrm{O}$ as our time axis. Using each such time axis one can define a hyperplane of events orthogonal to it, representing a set of simultaneous events, a "now". 5 If any notion of reality tracks simultaneity, it must accordingly be a notion that is also relative to choice of time axis.

Let me express this idea another way. Having chosen $\mathrm{O}$ and e in the initial argument, we observed that there is an event e' spacelike separated from both. For exactly one choice of time axis, the one in which e' lies in the hyperplane orthogonal to it, e' will have the same time coordinate as $\mathrm{O}$. If we think of $\mathrm{O}$ as the origin of some (inertial) coordinate system, then both $\mathrm{O}$ and $\mathrm{e}^{\prime}$ are assigned time $\mathrm{t}=0$. We are, however, free to choose amongst time axes that will assign to e' any positive or negative real number that we wish, however large, while still assigning $\mathrm{t}=0$ to $\mathrm{O}$. In light of this fact, what possible ontological significance could there be to the assignment to $e^{\prime}$ of the time $t=0$ ? How could the corresponding choice of time axis carry with it the "reality" of e'? As far as I can see, it cannot.

In presenting the original argument I wrote:

If two events happen at the same time, it would seem that it must be the case that if one is actual, determinate, or real (whatever these words mean), then the other must be actual, determinate, or real as well.

We can see now that this claim relies on resonances from our pre-relativistic understanding of its concepts for whatever plausibility it may possess.

\footnotetext{
${ }^{5}$ In addition to this relativity of simultaneity, it has been argued in effect that planes at other angles can equally well, though perhaps not equally simply or conveniently, represent nows. I discuss this view, the conventionality of simultaneity, in the first half of Savitt (2011)
} 
Moreover, even were it the case that simultaneity and reality went hand in glove, the original argument would fail because simultaneity in Minkowski spacetime is no longer transitive in the way that the original argument requires. In Newtonian spacetimes the binary relation ' $a$ is simultaneous with $b$ ' is transitive. That is, for any events $a, b$, and $\mathrm{c}$, if $\mathrm{a}$ is simultaneous with $\mathrm{b}$ and $\mathrm{b}$ is simultaneous with $\mathrm{c}$, then $\mathrm{a}$ is simultaneous with $\mathrm{c}$. In Minkowski spacetime this unique binary relation is replaced by the ternary relations ' $a$ is simultaneous with $\mathrm{b}$ in frame $\mathrm{F}$,' where ' $\mathrm{F}$ ' refers to an inertial frame determined by each of a non-denumerable infinity of time axes. Each of these three-place relations is transitive, but transitivity does not hold across frames. That is, if event $\mathrm{O}$ is simultaneous with event $\mathrm{e}^{\prime}$ in frame $\mathrm{F}$ and event $\mathrm{e}^{\prime}$ is simultaneous with event e in frame $\mathrm{F}^{\prime}$, it does not follow that $\mathrm{O}$ and e are simultaneous in any frame. In the example used in the original argument, it is clear that $\mathrm{O}$ and e are not simultaneous in any frame, if the two chosen events are timelike separated. So to repeat, even if there were some metaphysical tie between simultaneity and reality, transitivity could not transfer O's reality to e in the way that the original argument claims.

I have been talking about reality (or, at least, using the term 'reality') with a straight face in the previous paragraphs. I should not have been, but I did not want to distract the reader from the demonstrable infirmities of the original argument by making a more contentious claim. It is very difficult to know what is being claimed when it is asserted that the past or future are real, or are not real. Few authors stoop to explanation here. Putnam, to his credit, constrained his use of the predicate 'is real' with a set of postulates. Nevertheless, in a recent look back at the controversies initiated by his argument Putnam now believes that certain critics of the argument "are absolutely right, and... the question whether the past and future are "real' is a pseudo-question." (2008, 71)

The best way I know of to give content to questions about the reality of some class of objects is to follow the advice of John Austin (1962, chapter 7) - to specify the way or ways in which the objects of interest could fail to be real. With regard to the past and the future, it is by no means easy to specify ways in which they might fail to be real 
that leave one with an interesting or contentious question concerning their reality. ${ }^{6}$ Although this essay in general concerns ways in which pre-relativistic temporal notions may be reconstructed in relativistic spacetimes, it will not primarily address the traditional metaphysical questions about the reality of past, present, and future that were the main concern of RPPM, though these questions will be revisited in section $\S 3.6$. This essay will chiefly concern itself with finding relativistic counterparts of the present and their concomitant notions of temporal becoming.

\section{The Theorems of $\mathrm{SCH}$}

The positive criticism of the RPPM argument that I alluded to above is the definition of a relation of objective becoming in Minkowski spacetime, showing directly, if successful, that the conclusion of the RPPM argument is incorrect. This line of argument is much stronger than merely showing that the conclusion is not forced upon us by the RPPM argument. The basic idea of this positive argument is that what has become or is definite as of any spacetime point $\mathrm{x}$ (any "here-now") is that event itself plus any event along any possible timelike line through $\mathrm{x}$ but to the past of $\mathrm{x}$. If worldlines represent the histories of (possible) "observers", the idea is that any event that such a possible observer could have lived through prior to $\mathrm{x}$, as well as $\mathrm{x}$ itself, should be regarded as definite or as having become as of $\mathrm{x}$. More formally, the set of events that have become as of $\mathrm{x}$ are all points $\mathrm{y}$ in the spacetime such that there is a future-directed timelike curve from $y$ to $x^{7}$. This set of events or spacetime points is sometimes called the

\footnotetext{
${ }^{6}$ Exercise for the reader: try to find one.

${ }^{7}$ I will generally assume that timelike paths in spacetime are parameterized by proper time and refer to them as curves.
} 
chronological past of $\mathrm{x}$, and it is often denoted $\mathrm{I}^{-}(\mathrm{x}) .{ }^{8} \mathrm{I}^{-}(\mathrm{x})$ is in fact the set of points in the past light cone of $\mathrm{x}$.

This positive suggestion by Stein $(1968,1991)$ led to the formulation and proof of a theorem concerning objective becoming by him and generalizations by Clifton and Hogarth (1995) in two theorems. In this section, we will state these two results, following the presentation of Clifton and Hogarth but omitting their proofs, before we undertake to evaluate their philosophical significance in the remainder of this chapter.

The relation discussed both by RPPM and by SCH is a relation between points or events in, as already mentioned, time-oriented Minkowski spacetime, $<\mathrm{R}^{4}, \eta, \uparrow>$. ${ }^{9}$ One is allowed to use the Minkowski metric, $\eta$, and a chosen temporal orientation, $\uparrow$, in the definition of this relation in a four-dimensional real space, $\mathrm{R}^{4}$. Use of the metric amounts to use of what is known as causal structure, the light cones, in the definitions. (See Corollary 1(B) in Clifton \& Hogarth.)

The first theorem of Clifton and Hogarth concerns what they call absolute becoming, in contrast to the second, which concerns what they call worldline-dependent becoming. The difference between the two is that (as one might surmise from the terms) in the definition of worldline-dependent becoming one is permitted to choose an arbitrary inertial line (or time axis, as it was called in \$3.1) containing the point relative to which becoming is to be defined, whereas absolute becoming is relative only to choice of event (or spatiotemporal position). Since such a worldline is not definable from the causal structure or temporal orientation alone, one might suppose that this additional structure enables one to define more worldline-dependent becoming relations than absolute

\footnotetext{
${ }^{8}$ One can see that it is essential for this definition that an orientation has been chosen, so that one knows which is the past and which the future light cone at a point. It should also be clear that one can mutatis mutandis define the chronological future of a point $\mathrm{x}, \mathrm{I}^{+}(\mathrm{x})$.

${ }^{9}$ As we will see later, our way of skirting the $\mathrm{SCH}$ theorems is to define presents as regions rather than points and the becoming relation as holding between regions. I may make trivial changes in notation in my presentation of the $\mathrm{SCH}$ theorems in order to keep notation consistent between the various authors discussed, and I will use (trivially) altered notation in quotations as required.
} 
becoming relations. The surprise of the Clifton and Hogarth paper (contained in their second theorem) is that this conjecture is incorrect.

Much of the work in stating the theorems goes towards articulating conditions that an objective becoming relation should satisfy. The first condition imposed by Clifton and Hogarth is chronological becoming. If we let $\mathrm{B}(\mathrm{x})$ be the set of events that have become relative to event $\mathrm{x}$, then chronological becoming says that $\mathrm{I}^{-}(\mathrm{x}) \subseteq \mathrm{B}(\mathrm{x}){ }^{10}$

Chronological becoming is a natural enough assumption, but Clifton and Hogarth introduce some concepts used in the statement of their second theorem to justify the assumption of chronological becoming in their first theorem. First, for any event $\mathrm{x}$ in the spacetime, one can define $\mathrm{B}_{\gamma}(\mathrm{x})$ as the set of events that have become for $\mathrm{x}$, where $\mathrm{x}$ lies on a timelike curve $\gamma$. The role of the subscript ' $\gamma$ ' begins to become clear when one adds the assumption of worldline becoming, the assumption that if e lies on the curve $\gamma$ and is in the past light cone of $\mathrm{x}$, then $\mathrm{e}$ is in $\mathrm{B}_{\gamma}(\mathrm{x})$. More formally, worldline becoming requires that if $\mathrm{e} \in\left[\mathrm{I}^{-}(\mathrm{x}) \cap \gamma\right]$, then $\mathrm{e} \in \mathrm{B}_{\gamma}(\mathrm{x})$. They justify this assumption as follows (1995, 363):

The above assumption is... eminently reasonable. For an observer following any $\gamma$ will get at least a psychological sense that events she has actually lived through — or is living through — have become for her. And because the proponent of objective (albeit, worldlinedependent) becoming will not want to discount that psychological sense as just some figment of her imagination, he will surely want to

\footnotetext{
${ }^{10} \mathrm{It}$ is worth remarking that in addition to $\mathrm{I}^{-}(\mathrm{x})$ one typically distinguishes $\mathrm{J}^{-}(\mathrm{x})$, the causal past of $\mathrm{x} . \mathrm{J}^{-}(\mathrm{x})$ is the set of all events that lie either within or on the past lobe of the light cone at $\mathrm{x}$, or alternatively it is the set of all events y such that there is either a future-directed timelike or lightlike (or null) curve that connects $\mathrm{y}$ to $\mathrm{x}$. Then causal becoming is the condition that $\mathrm{J}^{-}(\mathrm{x}) \subseteq \mathrm{B}(\mathrm{x})$. Furthermore, in order to smooth the proofs of their theorems, Clifton and Hogarth stipulate that $\mathrm{x} \in \mathrm{I}^{-}(\mathrm{x})$ and $\mathrm{x} \in \mathrm{J}^{-}(\mathrm{x})$, so that the becoming relations are reflexive.
} 
account for it by incorporating it into the appropriate objective becoming relation $\mathrm{B}_{\gamma}$.

Worldline becoming is indeed a reasonable assumption, though we will eventually return to consider the subtle dissonance that one might discern in the claim, required by making the becoming relation reflexive, that an event that one is living through has become.

The second assumption made by Clifton and Hogarth with respect to worldlinedependent becoming is worldline transitivity. Suppose that $\mathrm{x}, \mathrm{y}$, and $\mathrm{z}$ are three events in the spacetime and $\gamma$ and $\gamma^{\prime}$ are two timelike curves. Then worldline transitivity requires that if $z \in B_{\gamma^{\prime}}(y)$ and $y \in B_{\gamma}(x)$, then $z \in B_{\gamma}(x)$. This condition enables an event $z$ not on $\gamma$ to have become for an event, such as $\mathrm{x}$, on $\gamma$. All such $\mathrm{z}$, however, will be in $\mathrm{I}^{-}(\mathrm{x})$, since worldline transitivity requires that there is a future-directed timelike curve from $\mathrm{z}$ to $\mathrm{y}$ and another future-directed timelike curve from y to x. Joining these two curves creates a future-directed timelike curve from $\mathrm{z}$ to $\mathrm{x}$.

Worldline transitivity might seem a weak condition, but it has two important implications. First, if $\gamma$ and $\gamma^{\prime}$ are not distinct curves, then worldline transitivity implies that worldline becoming is straightforwardly transitive along a given worldline. That is, if $\mathrm{z} \in \mathrm{B}_{\gamma}(\mathrm{y})$ and $\mathrm{y} \in \mathrm{B}_{\gamma}(\mathrm{x})$, then $\mathrm{z} \in \mathrm{B}_{\gamma}(\mathrm{x})$.

Second, if $\mathrm{x}$ and $\mathrm{y}$ are not distinct events, then, $\mathrm{z} \in \mathrm{B}_{\gamma^{\prime}}(\mathrm{y})$ if and only if $\mathrm{z} \in \mathrm{B}_{\gamma}(\mathrm{x})$. That is, according to Clifton and Hogarth, "worldline transitivity asserts that any two observers whose worldlines happen to cross at a point must agree, at least at that point, on what events have become real." $(1995,363)$ One can think of this agreement as indicating or reflecting a kind of objectivity in their becoming relation. What has become at a point in spacetime does not depend on the histories of any occupants of that point. We will return to this idea below.

Finally, Clifton and Hogarth $(1995,362)$ stipulate that a relation they call meshing holds between the two notions of becoming, absolute becoming and worldline becoming, 
that they have introduced. It is natural, they say, to suppose that if $z \in B_{\gamma}(x)$ for every timelike curve $\gamma$ through $\mathrm{x}$, then it should be the case that $\mathrm{z} \in \mathrm{B}(\mathrm{x})$, and conversely.

We can now state Clifton and Hogarth's results. First, we have a simple LEMMA: Consider the collection of worldline-dependent becoming relations associated with all worldlines (that is, all possible observers) in time-oriented Minkowski spacetime. If the collection satisfies worldline becoming and worldline transitivity, then chronological becoming must hold. (364).

Then following on the lemma is their version of Stein's theorem, THEOREM 1: Consider an absolute, worldline independent becoming relation in time-oriented Minkowski spacetime. If this becoming relation is objective, in that it is (implicitly) definable from time-oriented metrical relations and is transitive, and if this relation satisfies chronological becoming, then it can only be the relation of past chronological connectibility, or the relation of past causal connectibility, or the universal relation. (365)

The universal relation is the relation that $\mathrm{x}$ bears to every point $\mathrm{y}$ in the spacetime, where $y$ need not be distinct from $\mathrm{x}$, whereas the relation of past chronological connectibility is the relation $\mathrm{x}$ bears to every point in $\mathrm{I}^{-}(\mathrm{x})$ and the relation of past causal connectibility is the relation $\mathrm{x}$ bears to every point in $\mathrm{J}^{-}(\mathrm{x})$. Given the conditions of the theorem, then, one is left with just three choices as to what has become as of some spacetime point $\mathrm{x}$ - its chronological past, its causal past, or all of the spacetime.

In their second theorem Clifton and Hogarth explore the possibility that in addition to geometrical structure definable from the metric one be allowed to use a chosen timelike line containing a given point to determine what has become at that point. They call any becoming relation defined using this enlarged definitional base a worldlinedependent becoming relation. Are there more worldline-dependent becoming relations than there are worldline independent becoming relations?

One might think so. For instance, given an inertial line $\gamma$, one can construct the hyperplane of events orthogonal to it at any event $\mathrm{x}$ and one can then consider that set of 
events present for $\mathrm{x}$ (given $\gamma$ ). It is then natural to consider that set of events plus the union of their causal pasts to be what has become for $\mathrm{x}$ (given $\gamma$ ). This region of spacetime is not one of the three possible choices for what has become as of $x$ (on $\gamma$ ) in the first theorem above and so it might seem as if permitting one to choose a world line $\gamma$ enlarges the stock of possible presents.

This familiar and reassuring present faces a problem, however. Imagine a second inertial line $\gamma^{\prime}$ through the same point $\mathrm{x}$. If $\gamma^{\prime}$ is distinct from $\gamma$, then the hyperplane orthogonal to it will have only a two-dimensional hyperplane in common with the hyperplane orthogonal to $\gamma .{ }^{11}$ Two observers who reach $\mathrm{x}$ by travelling along different unaccelerated paths will then not agree as to what events have become. This disagreement in turn implies that the suggested version of the relation having become relative to an orthogonal hyperplane violates worldline transitivity. The conditions on becoming used in theorem 1, then, must be incompatible with this notion of becoming.

Just how much bite these conditions have we can see in the statement of Clifton and Hogarth's THEOREM 2 (371-72): Consider the collection of worldline dependent becoming relations associated with all worldlines (all possible observers) in time-oriented Minkowski spacetime. Suppose the collection satisfies worldline becoming and worldline transitivity, and that each relation in the collection is (implicitly) definable from timeoriented metrical relations and the relevant worldline for that relation. Then every becoming relation in the collection must be the relation of past chronological connectibility, or they all must be the relation of past causal connectibility, or they all must be the universal relation.

The upshot of this enormous effort can be stated succinctly. While Putnam claimed to have shown that the special theory of relativity rules out any notion of the passage of time or temporal becoming, the $\mathrm{SCH}$ theorems show that if any event $\mathrm{x}$ in

\footnotetext{
${ }^{11}$ If one considers only one temporal and one spatial dimension, as is usual when illustrating matters of principle in the special theory, then the two lines orthogonal to two distinct time axes have only the point of intersection of the time axes, $\mathrm{x}$ itself, in common.
} 
(time-oriented) Minkowski spacetime is taken to be "me-now", then there are two viable candidates for a relation of objective becoming, $\mathrm{B}(\mathrm{x})$, for that event, in addition to the universal relation. Either $\mathrm{B}(\mathrm{x})=\mathrm{I}^{-}(\mathrm{x})$ or $\mathrm{B}(\mathrm{x})=\mathrm{J}^{-}(\mathrm{x})$.

\section{A "No Go" Theorem?}

Thus far it seems as if SCH have won every battle against RPPM, but it has been claimed that they nevertheless have lost the war. That's the view of Simon Saunders (2000, S602), who wrote that "Clifton and Hogarth..., thinking that they were proving Stein right,.... have strengthened Putnam's point.” In another paper presented at the same Philosophy of Science Association symposium Craig Callender (2000, S592) wrote:

In particular quarters of this literature the idea that Stein conclusively refuted Putnam et al. and made becoming relativistically respectable seems to have achieved the status of conventional wisdom.... I must confess that, some quibbles aside, I've always found Putnam et al.'s argument eminently sensible. The idea is simply that any notion of becoming remotely similar to that found among advocates of the tensed view of time is not compatible with Minkowski spacetime.

While it is typically difficult to know precisely what philosophers mean by 'tense' when they use it to describe (a theory of) time itself rather than language, I think that in this case it's fair to say that a tensed theory is one that invokes a dynamic global now that divides all events into those that are present, past and future. It also seems reasonable to say that a single spacetime point or event dividing all events into those that have become for it and those that have not is not "remotely similar" to this global, tensed now. Callender is willing, however, to make the vague expression, "remotely similar," considerably more precise. 
Indicate the relation of becoming developed by $\mathrm{SCH}$ by Bxy, which is to be read: event or point $\mathrm{y}$ is definite (or has become) as of event or point $\mathrm{x}$. Then consider what Callender calls the non-uniqueness condition:

\section{(NU) $\exists x \exists y(B x y \&$ Byx \& $x \neq y)$}

NU, according to Callender (2000, S592), "merely says that at least one event in the universe shares its present with another event's present." 12 While the expression "shares its present" may also be subject to varying interpretation, I note that Callender later complains explicitly of Stein's present that it contains only a single point, ("According to this definition, only a single point is present for you, namely, your here-now." (S593)), and I take this uniqueness to be what primarily is denied (or is intended to be denied) in his non-uniqueness condition. It should be clear that anyone who subscribed to a tensed theory of time in the broad sense that I sketched just above would reject a present consisting only of a single event and so, presumably, would endorse NU.

It should also be clear on a little reflection that, if NU is satisfied, then the relation $B x y$ cannot be understood in terms of either $\mathrm{I}^{-}(\mathrm{x})$ or $\mathrm{J}^{-}(\mathrm{x})$. If $\mathrm{y} \in \mathrm{I}^{-}(\mathrm{x})$ and $\mathrm{y} \neq \mathrm{x}$, then $\mathrm{x} \notin$ $\mathrm{I}^{-}(\mathrm{y})$. Similarly for $\mathrm{J}^{-}(\mathrm{x})$. So if the relation of becoming satisfies the constraints that $\mathrm{SCH}$ place upon it and one wishes to have a present even "remotely similar" to that advocated by tensed time theorists (i.e., one that satisfies NU), then it would seem that the only option left, according to the theorems of SCH themselves, is the universal relation, just as Putnam claimed.

Callender formulates this result as a "No Go" Theorem.

For any binary relation $\mathrm{R}$ on time-oriented Minkowski spacetime, if $\mathrm{R}$ is $\mathrm{i}$ ) implicitly definable from time-oriented metrical relations, ii) transitive, iii) such that, if $y \in \mathrm{J}^{-}(\mathrm{x})$, then $\mathrm{Rxy}$, and iv) satisfies non-uniqueness, then $\mathrm{R}$ is the universal relation $\mathrm{U}$.

\footnotetext{
${ }^{12}$ According to Clifton and Hogarth (364) “"event a is coreal-or shares the same now-with event b' if and only if Bab and Bba."
} 
At first glance the No Go theorem seems to clinch the claim of Saunders and Callender that the very theorems of SCH (and the No Go theorem is, as Callender says, a straightforward corollary of these theorems) undermine Stein's claims and vindicate Putnam's.

Callender concludes that defenders of the present and becoming face a dilemma when they try to reconstruct temporal notions in the special theory of relativity. The traditional and philosophically interesting way of thinking of these notions, the way of tense, for which a proposition like NU holds true, "is provably inconsistent with Minkowski spacetime.” (S595) The way of SCH, on the other hand, yields counterparts-or rather, counterfeits--of the classical concepts that are so anemic that they appear to be "philosophically idle.” (S595)

Solid as this reasoning seems, it is the aim of the rest of this chapter to find a middle way, to introduce a notion of the present and of becoming in (time-oriented) Minkowski spacetime that does, in a way, satisfy NU and also satisfies the desiderata of $\mathrm{SCH}$ well enough to be called objective. But how, given the SCH theorems, can one find space to fit in a new notion of the present and becoming between the global view of tense and the austere constructions of SCH?

Perhaps the best first step towards opening up such space is to expand on the emphasized phrase "in a way" in the penultimate sentence. You may have noticed, five paragraphs above, that Callender's verbal translation of NU is not quite literal. What NU says literally is that there are two distinct events that have become for each other. What Callender says it says is that two distinct events share a present. These two ideas are distinct, even though typically linked. If, for instance, the present is a hyperplane of simultaneous, instantaneous events and the passage of time is the successive occurrence of such hyperplanes, then it should be clear that these hyperplanes consist of more than one event and that each event in a hyperplane has become for, or as of, every other event in the hyperplane. Conversely, in the SCH construction, the present is indeed just a single point and no two distinct events have mutually become. 
There is, however, at least one way in which these two ideas can be prised apart. While it is true that most traditional "tense" theorists think of (pre-relativistic!) time in terms of successively occurring hyperplanes of simultaneous point events, there is a minority stream in traditional thinking about time that holds the present to be temporally extended or "thick." This thick present is known as the specious or psychological present, the latter term because it is held that the perceived or psychological present is usually understood to include the time between what we remember as past and what we anticipate as future - an interval that has some small, finite (but not infinitesimal) duration.

In the classical case, a thick, global present consists of more than one point, and it is still plausible to maintain that simultaneous events in such a present have become for each other. But suppose one were to "thicken" a Stein point present in Minkowski spacetime to a segment of a worldline passing through the point, idealizing our human specious present. This relativistic thick present by definition no longer consists of a single point, and in that sense this present can be "shared" by pairs of distinct points in virtue of their being elements of or being contained in this present; but in such a present no pair of distinct points have mutually become and so do not literally satisfy NU.

This structure is less than completely satisfactory as a present in at least two ways. First, it is confined to a worldline and so has no spatial extent at all. Second, if two worldlines meet at a point and two such thick presents containing the point of intersection are specified along those two worldlines, then those two presents contain only that point in common. These are certainly unintuitive features for a present to have, but it may well be that no structure in Minkowski spacetime has all the intuitive features united in our common sense, pre-relativistic notion of the present. One reaction to this fact, if it is a fact, might be to claim that there is no relativistic counterpart of the present. A different, and I think ultimately more fruitful reaction, would be to claim that although there is no unique relativistic counterpart of the present, there are various structures in Minkowski spacetime that have some of the features of the pre-relativistic present. Although it may be that no structure has all the features that characterize the pre-relativistic present, each relativistic candidate has enough of those features to be considered $a$ present. The 
question: what is the present in Minkowski spacetime? is better resolved into the questions: If one is to search for a relativistic successor concept to the commonsense notion of the present, what structures in Minkowski spacetime can play the role of the pre-relativistic present and how well do they do it?

We know that pre-relativistic time bifurcates in the special theory into coordinate time and proper time. Why should it not be that other temporal notions divide as well? If so, then one has simply to weigh the merits of the various candidates, recognizing in advance the possibility that no one candidate will be entirely successful.

\section{Local Presents.}

In light of the discussion of the last section, let us begin our search for possible successor structures to the commonsense present in Minkowski spacetime by relaxing the assumption that presents must be instantaneous. We could then define a structure that is dependent on a choice of worldline $\gamma$, making our new structure world line dependent in the sense of Clifton and Hogarth, and also dependent on the choice of an interval along $\gamma$. We might, for instance, choose a closed interval of points $[a, b]$ on $\gamma$, where our conventions will be that $\mathrm{a} \neq \mathrm{b}$ and that $\mathrm{a}$ is to the past of $\mathrm{b}$. We can then, as we have noted, use causal structure like $\mathrm{I}^{-}(\mathrm{x})$ or $\mathrm{J}^{-}(\mathrm{x})$ - or for that matter, though they have had no role in our deliberations so far, $\mathrm{I}^{+}(\mathrm{x})$ or $\mathrm{J}^{+}(\mathrm{x})$ - to define further structures.

Turn now to a line of thought found in section V of Stein (1991). ${ }^{13}$

\footnotetext{
13 But briefly touched on in footnote 14 of Stein $(1968,15)$ : “The paradox is mitigated when we observe that, for processes of more than instantaneous duration, a meaningful and intuitively satisfying notion of "contemporaneity" can be defined: two such processes may be said to be contemporaneous if part of each is past to part of the other-in other words, if mutual influence ("communication") is possible between them.
} 
Let us consider a "specious present" $\pi$ of some percipient being; and let us call an event $e$ "contemporaneous" with $\pi$ if signalsinteraction - influence — can occur mutually between $e$ and $\pi$... [T] he set of events contemporaneous with a specious present will always be a spatially extended one. And it is, I think, of very great relevance... that this spatial extent—although finite — is in fact and in principle, as a matter of physics, always, in a certain sense, immensely large. (159)

We can represent a specious present $\pi$ as a set of points [a,b] of appropriate duration $\tau$ on some world line $\gamma$. What sent of points is contemporaneous, in Stein's sense, with $\pi$ ?

In the first instance, let us confine ourselves to causal interactions represented by timelike worldlines. ${ }^{14}$ The events that can influence events in $\pi$ are just the events in the past light cones of events in $\pi$. The set containing all events that can act upon any event in $\pi$ must be the past light cone of the latest event in $\pi$, $\mathrm{I}^{-}(\mathrm{b})$. Similarly, the events that can be acted upon by any event in $\pi$ are the events in the future light cones of events in $\pi$. The set containing all such events is the future light cone of the earliest event in $\pi, \mathrm{I}^{+}(\mathrm{a})$. It follows that all events that can interact with events in $\pi$ must be in both sets - that is, these events must be in the intersection of the two sets, $\mathrm{I}^{+}(\mathrm{a}) \cap \mathrm{I}^{-}(\mathrm{b})$.

Let me call this set, the intersection of two light cones, $P_{\gamma}(a, b)$ in order to highlight its dependence on two events, $a$ and $b$, lying on some timelike curve $\gamma$. Since these events lie on a curve there will be some proper time interval $\tau$ between the two, the time indicated by an ideal clock following that curve, with the proper time in general

\footnotetext{
${ }^{14}$ It will be easy to extend the discussion to include null curves.
} 
depending on the chosen curve. ${ }^{15}$ If the curve connecting two events is an inertial line (or, more generally, a geodesic), then the proper time between them is maximal. ${ }^{16}$

$\mathrm{P}_{\gamma}(\mathrm{a}, \mathrm{b})$ is the intersection of the interiors of two light cones - the future light cone of $\mathrm{a}$ and the past light cone of $\mathrm{b}$ - and has sometimes accordingly been called a double cone. ${ }^{17}$ These regions are open sets of the Alexandrov topology. (Penrose, 1972) They are sets with an interesting physical motivation, since they are sets of all events that can interact with a "thick" specious present (so one might by way of contrast call them "fat" presents). They are of some interest in mathematics and physics, as just noted, but what might have they to do with the present and becoming? To see the connection, it would be helpful at this point to step back and look at some general issues.

RPPM and SCH were arguing about whether certain notions, familiar to commonsense and partially manifest in ordinary experience, are to be found, possibly in attenuated or altered but nevertheless still recognizable form, in Minkowski spacetime. If the commonsense present is a global hyperplane of simultaneity, then such hyperplanes are to be found in Minkowski spacetime, but they exist there in such profusion that they can no longer bear their pre-relativistic significance (as pointed out in chapter 2) and fail to be "objective" (as pointed out earlier in this chapter).

As a response to the well-known problems with global hyperplanes as presents in Minkowski spacetime, along with the new problem with point presents signaled by the no-go theorem, we suggested that presents have some duration. But just how much duration? Before trying to answer that question directly, let us beat about the neighboring bushes to consider a bit of the everyday behaviour of the indexical terms 'now' and

\footnotetext{
${ }^{15}$ See Arthur (2010) for a discussion of the clock hypothesis.

${ }^{16}$ Curves representing the paths of accelerated "observers" are longer in spacetime diagrams than the straight lines representing the paths of inertial observers, but the proper time along such "longer" paths is less than the proper time on the paths of inertial observers (between the same two spacetime points). This fact underlies the so-called twin paradox.

${ }^{17}$ See Halvorson (2007), p. $740 \mathrm{ff}$, in which the double cone is used as a convenient structure on which to base the development of algebraic quantum field theory.
} 
'here'. 'Here' in its primary use picks out the spatial location of the speaker (assuming for simplicity that the word is spoken), but just how extensive a location is intended is just as much a matter of context as which location it is. I might, for instance, from time to time mean 'here' to indicate my home, Vancouver, Canada, Earth, or... The extent of 'now' is similarly elastic in its function of picking out the temporal locations. If I say "Public telephones once were very common, but now they are very hard to find", I am clearly not indicating a point present. Sometime in the last decade or so, public telephones became noticeably hard to find. And if I say, "There are no dinosaurs now, but once they were common", the period of time indicated by 'now' is even longer.

If 'now' is meant to indicate what the speaker regards in a given context as the present time, then one has to realize that there will be an ineluctable tension between the flexible use of this term in everyday life and the precise geometric tools one has to hand to fashion a relativistic counterpart. I think one can partially bridge this gap by recognizing that there is a kind of central or core use of 'now' to indicate time that one takes to be neither past nor future, neither remembered nor anticipated--the specious or the psychological present. This specious present is quite variable, both inter-personally and intra-personally, but typical bounds for it are from .3 seconds to 3 seconds.

If one chooses values of $\tau$ that are in the interval [. $3 \mathrm{sec}, 3 \mathrm{sec}$ ], then the stretches of proper time $\tau$ along a timelike worldline $\gamma$ that limit the choice of intervals $[a, b]$ on inertial worldlines and which then anchor the double cone or "fat" regions introduced above would correspond to (possible) psychological presents of some observer. This correspondence does not entail that such regions are "subjective" or mind-dependent. While the scale for $\tau$ is set by contingent features of human experience, given that scale there need be no humans actually occupying a timelike worldline $\gamma$ for an appropriate stretch of it to be a thick present $[\mathrm{a}, \mathrm{b}]$ or for a region to be its corresponding fat present $\mathrm{P}_{\gamma}(\mathrm{a}, \mathrm{b})$. There were such stretches of worldlines and regions of spacetime before humans existed, and there will be such after we cease to exist. These stretches and regions exist in parts of spacetime where humans have never gone and will never go. 
Having recognized that the scale employed is somewhat vague, let me further suggest that no philosophical issue is begged, though exposition is greatly simplified, if we simply choose one specific value in the range of specious presents to be the common extent or duration of all presents. The obvious one to choose is a middling value that is easy to calculate with, like 1 second. I take these simplifications as analogous to the kind of simplification one employs in presenting the special theory of relativity when one restricts discussion to inertial frames in "standard configuration". The discussion is not completely general, but no essential idea is lost is lost in the simplification, no question begged.

The idea is then to move from the observation that the proper time interval $[\mathrm{a}, \mathrm{b}]$ of length $\tau$ in the interval $[.3 \mathrm{sec}, 3 \mathrm{sec}]$ represents a specious present of a possible inertial observer to the claim that 1 second is a useful, albeit arbitrarily chosen, value in this interval and then finally to the claim that $\mathrm{P}_{\gamma}(\mathrm{a}, \mathrm{b})$, with $\gamma$ the worldline of an inertial observer and the events a and b separated by a proper time of 1 second, is a viable relativistic counterpart of the commonsense present.

The first thing we note in defense of this claim is the minimal and obvious ${ }^{18}$ fact that two distinct events can share such a present in a sense indicated near the end of section 3.3. Two distinct events e and $\mathrm{e}^{\prime}$ share a present $\mathrm{P}_{\gamma}(\mathrm{a}, \mathrm{b})$ if $\mathrm{e} \neq \mathrm{e}^{\prime}$ and $\mathrm{e} \in \mathrm{P}_{\gamma}(\mathrm{a}, \mathrm{b})$ and $\mathrm{e}^{\prime} \in \mathrm{P}_{\gamma}(\mathrm{a}, \mathrm{b})$. In this feature $\mathrm{P}_{\gamma}(\mathrm{a}, \mathrm{b})$ differs from the point presents considered above. It is easy to see that we may choose events e and $\mathrm{e}^{\prime}$ in $\mathrm{P}_{\gamma}(\mathrm{a}, \mathrm{b})$ that are spacelike separated. In that case $\mathrm{e} \notin \mathrm{I}^{-}\left(\mathrm{e}^{\prime}\right)$ and $\mathrm{e}^{\prime} \notin \mathrm{I}^{-}(\mathrm{e})$. There is no temptation, then, to think that either event has become with respect to the other, and so there is no link in this case between being co-present and mutual becoming.

While $\mathrm{P}_{\gamma}(\mathrm{a}, \mathrm{b})$, unlike a point present, is extended through space, it is not extended through all space, like a hyperplane of simultaneity. For example, if we use our

\footnotetext{
${ }^{18}$ Obvious because the interval $[\mathrm{a}, \mathrm{b}]$ on $\gamma$ contains more than one point, and $\mathrm{P}_{\gamma}(\mathrm{a}, \mathrm{b})$ contains that interval.
} 
representative value of $\tau=1$ second, then the greatest extent of $\mathrm{P}_{\gamma}(\mathrm{a}, \mathrm{b})$ is $300,000 \mathrm{~km}$ at its waist, where the two light cones meet. While this distance is large —even "immensely large" as Stein put it_compared to everyday distances, our presents are nevertheless local structures rather than global. It is puzzling to think of time as a local phenomenon, but every way of introducing temporal notions into Minkowski spacetime has its own puzzles when compared to the pre-relativistic concepts.

The great size of these local presents enables them to some degree to evade an objection that Callender, following Putnam, makes against point presents. Callender writes:

[O]ne's past is not the union of one's former nows on this view.

Consider the Yankees winning the World Series in NY in 1998.

Since I wasn't in NY at that time, and yet the event is now in my backward light cone, the Yankee's win determinately happened for me but at no time was it present for me. (S594)

This objection does indicate one way in which a point present is unlike the pre-relativistic global present, but it is not so straightforwardly a point of difference for fat presents. Assuming that Callender's world line has stayed close to the surface of the earth, in 1998 he did share a series of local presents with the events of the World Series. Of course, for distant enough events it is still true that they can enter one's objective past without ever being present, but one might find this oddity less disconcerting for events on the moons of Jupiter or in another galaxy than for events in Sydney.

\section{Becoming}

If one were to consider $\mathrm{P}_{\gamma}(\mathrm{a}, \mathrm{b})$ with $\tau=1$ a reasonable candidate for a present in Minkowski spacetime, the next items on the agenda should be to define a notion of 
becoming with respect to it and to see to what extent this notion of becoming can said to be objective in the sense of satisfying constraints on becoming relations like those imposed in the $\mathrm{SCH}$ theorems.

The first, general, and quite natural, way to think of becoming in this context is to say that a set of events or region $\beta$ has become for a region $\alpha$ if and only if for every $\mathrm{y} \in \beta$, there is some $\mathrm{x} \in \alpha$ such that $\mathrm{y} \in \mathrm{I}^{-}(\mathrm{x}) .{ }^{19}$ The largest region that has become for $\alpha$, then, is the union of the past light cones of all events in it. For the fat, local presents we have been considering, we have the nice result that the largest region that has become for $\mathrm{P}_{\gamma}(\mathrm{a}, \mathrm{b})$ is $\mathrm{I}^{-}(\mathrm{b})$. We could indicate this by writing $\mathrm{B}\left(\mathrm{P}_{\gamma}(\mathrm{a}, \mathrm{b})\right)=\mathrm{I}^{-}(\mathrm{b})$ Since $\mathrm{P}_{\gamma}(\mathrm{a}, \mathrm{b}) \subset$ $\mathrm{I}^{-}(\mathrm{b}), \mathrm{P}_{\gamma}(\mathrm{a}, \mathrm{b})$ has become (or, better, is definite) as of itself, according to this definition.

We can also imagine a slight variant of this definition. When the becoming relation held between pairs of events, $\mathrm{SCH}$ required the relation to be reflexive, but I think it was for them a "don't care" case. They offered no compelling reason for or against reflexivity; it was just convenient for them to assume it. When the relation of becoming holds between regions of spacetime, there might seem to be a bit more difference. Recall the oddity noted above of saying that events that one is living through or experiencing (in a specious present) have become. ${ }^{20}$ If one is impressed by this sort of oddity, then one would assume that a region has not become as of itself. That is, let us suppose that that relation of becoming when it holds between regions is not reflexive. Then the formal definition of becoming is the same as that given above, except that we exclude any events in the region $\beta$ that are also in the region $\alpha$. If we write $\mathrm{B}^{*}(\alpha)$ to indicate the region $\beta$ that has become for region $\alpha$ when the underlying relations are irreflexive, we then say that

$$
\mathrm{B}^{*}(\alpha)=\left\{\mathrm{y}: \mathrm{x} \in \alpha \& \mathrm{y} \in \mathrm{I}^{-}(\mathrm{x})\right\}-\alpha
$$

\footnotetext{
19 Following the definition of region $\beta$ is definite as of region $\alpha$ in Myrvold $(2003,478)$.

${ }^{20}$ There is a long history of reflection on the ontological status of the specious present that might be helpful here. See Andersen \& Grush.
} 
Since these region-relative becoming relations are different from any of those admitted by the $\mathrm{SCH}$ theorems, the suspicion might then arise that somehow they fail to meet a condition that a bona fide becoming relation should satisfy.

The condition of chronological becoming (the first condition imposed by Clifton and Hogarth) required that $\mathrm{I}^{-}(\mathrm{x}) \subseteq \mathrm{B}(\mathrm{x})$, where $\mathrm{B}(\mathrm{x})$ is the set of points that have become for $\mathrm{x}$. The intuition behind this requirement, you may recall, is that any event that could effect $\mathrm{x}$ should be in $\mathrm{B}(\mathrm{x})$. To extend this to regions one would require that, if every event in some region $\beta$ can effect some event or other in a region $\alpha$, then $\beta$ must be a subset of $\mathrm{B}(\alpha)$. This condition is guaranteed by the definition of region-relative becoming above. ${ }^{21}$

Another constraint on becoming in the $\mathrm{SCH}$ theorems is transitivity. In our case, transitivity would take the following form: if $\alpha, \beta$ and $\delta$ are regions of Minkowski spacetime, then if $\delta \subseteq \mathrm{B}(\beta)$ and $\beta \subseteq \mathrm{B}(\alpha)$, then $\delta \subseteq \mathrm{B}(\alpha)$. Since the relation being in the past light cone of is transitive (That is, if $\mathrm{z} \in \mathrm{I}^{-}(\mathrm{y})$ and $\mathrm{y} \in \mathrm{I}^{-}(\mathrm{x})$, then $\mathrm{z} \in \mathrm{I}^{-}(\mathrm{x})$ ). , then the becoming relation between regions must be transitive as well. Since transitivity holds for regions in general, it will hold a fortiori for local presents.

We should recall here, however, that the transitivity of absolute becoming entailed that two observers who met at a point agreed as to what has become (as of that point). That agreement is lost for region-relative becoming as construed above. Suppose that two observers, $\mathrm{O}$ and $\mathrm{O}^{\prime}$ on distinct inertial worldlines $\gamma$ and $\gamma^{\prime}$ meet at some event $\mathrm{e}$ and that we use our default value of 1 second for $\tau$ to define their local presents. Then if $a$ and $b$ are events $1 / 2$ second before and $1 / 2$ second after e on $\gamma$ and if $a^{\prime}$ and $b^{\prime}$ are the events $1 / 2$ second before and $1 / 2$ second after e on $\gamma^{\prime}$, we can see that $a \neq a^{\prime}$ and $b \neq b^{\prime}$. It then follows that $\mathrm{P}_{\gamma}(\mathrm{a}, \mathrm{b}) \neq \mathrm{P}_{\gamma^{\prime}}\left(\mathrm{a}^{\prime}, \mathrm{b}^{\prime}\right)$, that $\mathrm{I}^{-}(\mathrm{b}) \neq \mathrm{I}^{-}\left(\mathrm{b}^{\prime}\right)$, and hence that $\mathrm{B}\left[\mathrm{P}_{\gamma}(\mathrm{a}, \mathrm{b})\right] \neq$ $\mathrm{B}\left[\mathrm{P}_{\gamma^{\prime}}\left(\mathrm{a}^{\prime}, \mathrm{b}^{\prime}\right)\right]$

\footnotetext{
${ }^{21}$ For $\mathrm{B}^{*}(\alpha)$, we do not quite have all events that can effect events in region $\alpha$; the events in $\alpha$ itself are absent.
} 
However, when the relative speed of two observers is small compared to the speed of light, the difference between these two local presents is nearly insignificant. As I have noted elsewhere, if the relative speeds of $\mathrm{O}$ and $\mathrm{O}^{\prime}$ were $4 \mathrm{~km} / \mathrm{h}$ (say, $\mathrm{O}$ walked past a stationary $\mathrm{O}^{\prime}$ ), then the difference between the volumes of their two local presents (with $\tau=1$, of course) would amount to about one half of one millionth of 1 per cent $\left(\sim 5 \times 10^{-9}\right) .^{22}$ The extent of this overlap must surely be part of the explanation of the prerelativistic belief that we share a common present.

Finally, Clifton and Hogarth require that a bona fide worldline-dependent becoming relation be definable from the metric, a temporal orientation, and a worldline. The only element we have added to this is a specified interval on the given worldline, but these intervals are given in terms of proper time and proper time is explicitly definable in terms of the metric (as we saw in chapter 2). Put another way, these intervals are preserved under all time-oriented metrical automorphisms that preserve chosen worldlines, the maps $\phi_{\lambda}$ that Clifton and Hogarth use in the proof of their second theorem.

What other sets are definable in this way, given that one is permitted choose an interval on a worldline? As far as I can see, the question is open. Perhaps this expansion of the definitional base opens up what Clifton and Hogarth call a "Pandora's Box" of other structures. If so, I will leave it to others to define them and to provide motivation for them, if they believe that any of them are viable alternative candidates in the field of successors to the pre-relativistic present.

\section{Time and Reality}

Let us step back from the details of the preceding arguments to ensure that two important points are clear. We began this chapter by considering a generic argument that

\footnotetext{
${ }^{22}$ See (Savitt 2009), which contains further arguments in defense of the local present. I am grateful to my student Alexandre Korolev for the calculation.
} 
would imply, amongst other things, the falsity of the metaphysical view that Putnam called presentism:

(P) All (and only) things that exist now are real.

We have argued against RPPM that there are coherent, relativistic notions of the present — an interval $[\mathrm{a}, \mathrm{b}]$ on a timelike worldline $\gamma$ and our local present, $\mathrm{P}_{\gamma}(\mathrm{a}, \mathrm{b})$---that are more than a single spacetime point or event but are not so broad as a global hyperplane or global achronal surface. We have also argued that there is a coherent notion of becoming, or at least of having become, relative to such presents. Philosophers will be well aware, moreover, that since the time that RPPM wrote, the metaphysics of time has become a "hot" area in analytic metaphysics, with many philosophers busily articulating or vociferously defending versions of presentism. Recently, for instance, Hestevold (2008) described a new version of presentism featuring a present of some small duration-thick presentism. It would not be unreasonable to suspect that we too are scrambling aboard this bandwagon.

While not unreasonable, however, the first important point is that this suspicion would be completely incorrect. We are not thick presentists, or presentists of any sort. The general reason we wish to distance ourselves from this view as well as from its supposed opposite, eternalism--the view that "all events are equally real"--is that in our opinion neither view has been stated in a way that is both clear or unambiguous and also significant or non-trivial.

It sometimes seems as if the debate between presentists and eternalists is a particularly heated dispute over whether or not to apply the empty honorific term 'real' to various spacetime points or regions. If this is an accurate characterization, then it is clearly of no interest which side in the dispute prevails. The way to give the term 'real' content, as suggested in section 1 of this chapter, is to heed Austin's maxim by specifying a clear contrast term, a way in which the entities in question could fail to be real. It is not easy, however, to specify such a contrast in a way that makes the debate between presentists and eternalists significant. 
Suppose, for instance, that something fails to be real by being non-present. Then presentists are speaking truly when they say that only the present is real, but the truth they utter is a vacuous or trivial truth. Of course the past and future, being non-present, fail to be real, given that understanding of 'real'.

On the other hand, suppose that something could fail to be real by having no place at all in the spacetime network, like most fictional objects (Sherlock Holmes) or abstract entities (numbers, propositions). Then of course past, present and future events and entities are all equally real, given that understanding of 'real'.

We suggest that it is not easy to find an intermediate specification of the way something could fail to be real that gives content to the alleged controversy. In addition, this vagueness feeds into or off of another unwanted degree of freedom in the specification of the two views, a peculiar sort of ambiguity or polysemy in the term 'exist'. In particular 'exist' may be either a present tense verb or something else, typically called a tenseless verb. In the latter case, it may be thought of as the disjunction of three clauses. Some thing $\mathrm{x}$ exists (tenselessly) if either $\mathrm{x}$ did exist, does exist, or will exist. Of course, when 'exist' is used as a present tense verb, then it is true (but trivially so) to say that only presently existing things exist. On the other hand, it is also true, but equally trivially, that all past, present and future things or events exist (tenselessly). No one could disagree with either assertion, and so they represent no significant controversy.

It is easy to exploit the vagueness and to compound the ambiguity. It is tempting to try to sidestep the problem by changing terms (the presentist, it might be said, in some way "ontologically privileges" the present), as if the new claim were clearer than the old one. We will not attempt to examine, or even enumerate, all the twists and turns in this set of arguments, ${ }^{23}$ but it might be useful to look at one instructive case. ${ }^{24}$ Consider the following attempt by Christian Wüthrich (forthcoming) to characterize a substantive presentism/eternalism dispute, where $\mathcal{M}$ is understood to be "a four-dimensional manifold with certain topological and differential structure":

\footnotetext{
${ }^{23}$ As I tried to do in Savitt (2006).

${ }^{24}$ I am indebted to Christian Wüthrich for making his paper available to me prior to publication.
} 
[E]ternalism can be understood as the position awarding existence to all events in $\mathcal{M}$, with the spatio-temporal properties given by the relations among the events as they are encoded in the metric field $g_{a b}$ defined on $\mathcal{M}$. Presentism, on the other hand, takes an equivalence relation $S$ which foliates $\mathcal{M}$ ("simultaneity") and then restricts physical existence to those events in the folium corresponding to "now". Time, for the presentist, then is the onedimensional linearly ordered quotient set induced by $S$. As can be seen from these formulations, presentism and eternalism have a substantive disagreement. Their respective sets of existing spacetime events are simply non-identical in that the presentist's is a proper subset of the eternalist's. Furthermore, presentism requires a well-defined equivalence relation, but eternalism does not. $(\S 3)$

Let me begin with a limited expression of agreement with some of what Wüthrich says. Self-identified presentists rarely, if ever, say what they take the present to be. One suspects, along with Wüthrich, that the present they are defending is (tacitly) a global hyperplane of simultaneous events and that indeed for them time is the quotient set of spacetime under the relation $S$. But notice then that Wüthrich understands presentists to be claiming that spacetime has the structure of Aristotelian or Galilean spacetime ${ }^{25}$ rather then the structure of Minkowski spacetime or some general relativistic spacetime. This claim is not a claim in metaphysics, but rather it is a claim in physics, and I will happily agree with him that it is almost certainly incorrect.

One can use words as one wishes, and if this is what Wüthrich means by presentism, then I will have to agree that it is a substantive claim and likely false. But to see that the issue spotlighted here is not the core or central issue, all one need do is note that the opposite of presentism in this sense is not eternalism, since Wüthrich has

\footnotetext{
${ }^{25}$ As these terms are explained in chapters 1 to 4 of Geroch (1978).
} 
presentists and their opponents making conflicting claims about the structure of spacetime (Aristotelian or Galilean vs. relativistic). Wüthrich has pointed to a difference, but it's not the fundamental or central difference

The presentist/eternalist dispute is generally held to be a disagreement about ontology rather than geometry, ${ }^{26}$ and we move closer to the way it is usually understood in the philosophical literature when Wüthrich writes that the "respective sets of existing spacetime events are simply non-identical in that the presentist's is a proper subset of the eternalist's." Let us concentrate, then, on this attempt at a core characterization of the pair of views, rather than on the peripheral contrast I just highlighted so that we may put that red herring clearly and firmly aside.

Those with a skeptical attitude towards the presentist/eternalist dispute insist that, if the dispute is genuine, then it ought to be possible to exhibit one clear, unambiguous assertion affirmed by one side and denied by the other. In the sentence from Wüthrich's characterization of the two sides just quoted above, we find the word 'existing'. This is a present participle of the verb 'exist', and the skeptic immediately reminds us that this can be either a tensed or a tenseless verb. Which is it in Wüthrich's case?

Suppose that the verb is tensed. Then ' $\mathrm{x}$ exists' is roughly equivalent to ' $\mathrm{x}$ exists now' or ' $x$ currently exists' or ' $x$ presently exists'. Since presentists 'restrict physical existence to those events in the folium corresponding to "now", they restrict the things that (physically) exist, that is, the "existing spacetime events"--that is, the spacetime events that exist now--to those events that exist now. Lives there someone who wishes to deny that events that exist now exist now?

Suppose that the verb is detensed. Then ' $\mathrm{x}$ exists' is roughly equivalent to ' $\mathrm{x}$ existed or $\mathrm{x}$ currently exists or $\mathrm{x}$ will exist'. If $\mathrm{x}$ is an event, we could paraphrase ' $\mathrm{x}$ exists' as 'x occurs somewhere in the spacetime manifold $\mathcal{M}$ '. Since eternalists are given to "awarding existence to all events in $\mathcal{M}$ " and $\mathcal{M}$ is an entire four-dimensional spacetime

\footnotetext{
${ }^{26}$ Wüthrich seems to look at it this way was as well. Earlier in $\S 2$ he writes, "presentism... is purely a claim concerning existence." Then he repeats: "Presentism is only an ontological claim concerning existence, not one about any further properties of that which exists (over and above that it is present)."
} 
manifold, then eternalists are those who say that any event past, present or future "exists"- that is, is past, present or future. Lives there someone who wishes to deny this?

One just might suppose that a "presentist" is willing to deny this by asserting that no past events have ever happened and no future events ever will. There is just the present. But I would be hesitant to interpret someone as advancing this wild claim, and I believe that Wüthrich would be even more hesitant than I. In the first paragraph of his paper he writes, "Presentism derives its appeal, first and foremost, from our intuition that past things have existed, but no longer do, and that future events have yet to occur." Wüthrich would not attribute to presentists a claim that flatly denied the intuition from which he believes their view derives its appeal. ${ }^{27}$

Possibly there is some other way to understand 'exist', a way that makes the dispute significant. If so, then it's up to someone who believes that the dispute is significant to spell this understanding out. Suffice it to say that, until that task is done, we are sympathetic to the claim of the skeptics that no significant metaphysical controversy has yet been defined or demarcated in this area.

At this point the question might arise: if our notions of the present and becoming are not fraught with metaphysical significance, what significance do they have? This question raises in turn the question, what significance do the investigations of this chapter have? In fact, what is the general nature of these investigations? A convenient way to get a handle on these questions was provided in a recent paper by Callender (2008). I am going to quote the opening paragraph, where by "manifest image" Callender means our commonsense view of the world and its contents and by "scientific image" an idealized, unified scientific world-picture.

The manifest image is teeming with activity. Objects are booming and buzzing by, changing their locations and properties, vivid perceptions are replaced, and we seem to be inexorably slipping into the future. Time- or at least our experience in time-

\footnotetext{
${ }^{27}$ And, by the way, are eternalists supposed to deny this intuition that "past things have existed, but no longer do, and that future events have yet to occur?"
} 
seems a very turbulent sort of thing. By contrast, time in the scientific image seems very still. The fundamental laws of physics don't differentiate between past and future, nor do they pick out a present moment that flows. Except for a minus sign in the relativistic metric, there are few differences between the temporal and spatial coordinates in natural science. We seem to have, to echo another debate, an "explanatory gap" between time as we find it in experience and as we find it in science. Reconciling these two images of the world is the principal goal of philosophy of time.

Some readers will recall that this call to reconcile the manifest and scientific images is the program enunciated in Sellars (1963), a profound (at least in my view) project that he proposed at a time when the dominant metaphilosophical paradigm was that the task of philosophy was to "show the fly the way out of the fly-bottle" by reminding us of the subtleties of everyday discourse.

Callender, as the title of his paper suggests, focuses on the question: Why is it that we take ourselves to be inhabiting a common or shared present? His explanation, relying on some insights of Jeremy Butterfield (1984) and the results of recent experiments in time perception, is that there are human cognitive mechanisms that construct from the manifold of experience an integrated present. The working of these mechanisms requires that this integrated present be extended in time. Corresponding to extended presents are "present patches", "spatiotemporal region[s] over which typical observers in typical environments do not require a time stamp in order to reliably navigate their environments." $(2008,349)$ These present patches, presumably, are large enough for us to share.

One might see the arguments of the earlier sections of this chapter as, at least in part, addressing the same question as Callender but working from spacetime geometry to an extended present rather than from psychological mechanisms to an extended present. (Working from the outside in, as it were, rather than from the inside out.) We do meet at an extended present, but Callender thinks his present patches are mind-dependent, 
whereas I take local presents to be objective spacetime regions (but scaled to human proportions). If we can find a way to bridge this gap, if present patches can be made precise as spacetime regions of the form $\mathrm{P}_{\gamma}(\mathrm{a}, \mathrm{b})$, then he and we have put together the beginnings of a picture connecting experience to spacetime, and spacetime to experience, in the spirit of the Sellarsian program sketched above

I do not wish to overstate the case as to what has been accomplished here. It is difficult to accommodate our pre-relativistic or manifest ideas of the present and the passage of time to the geometries of relativistic spacetimes. Perhaps one should just (somehow!) adjust one's old views to new theoretical perspectives, but even so radical a thinker as Einstein was troubled by the magnitude of the adjustment to temporal concepts required by relativity. Here is a famous report of a conversation Einstein had with Rudolf Carnap $(1963,37)$ :

Once Einstein said that the problem of the Now worried him seriously. He explained that the experience of the Now means something special for man, something essentially different from the past and the future, but that this important difference does not and cannot occur within physics. That this experience cannot be grasped by science seemed to him a matter of painful but inevitable resignation.

On our view 'now' is an indexical term that indicates one's temporal location, just as 'here' indicates one's spatial location. In Minkowski spacetime regions like $\mathrm{P}_{\gamma}(\mathrm{a}, \mathrm{b})$ are just as available to locate one temporally as hyperplanes of simultaneity were (thought to be) available for that purpose in pre-relativistic spacetime. In this guise, the now (or the present) is needed in physics no more (or no less) than the here is.

There is a satisfying corollary of our view that is worth noting. In discussions of time in which the existence of global presents is taken for granted, it has been remarked that there is an asymmetry between the uses of the indexicals 'now' and 'here'. It matters when one uses 'here', but it does not matter where one uses 'now'. It is sometimes 
inferred from this asymmetry that time is more fundamental than space. In our account of the local present in Minkowski spacetime, however, 'now' and 'here' have a reciprocal, symmetric dependence. One can then infer nothing as to whether time is more fundamental than space (or vice versa), which is as it should be if spacetime is fundamental.

Not only is the now part of physics (as a region of a relativistic spacetime), so also is the passage of time. Kurt Gödel captured the basic pre-relativistic idea of the passage of time in one elegant sentence when he wrote $(1949,558)$

The existence of an objective lapse of time... means (or, at least, is equivalent to the fact) that reality consists of an infinity of layers of "now" which come into existence successively.

By a "layer of "now" I take Gödel to mean a global hyperplane of simultaneous events. To say that they come into existence successively is to say that these sets of events happen one after another. I have argued that this way of thinking about the passage of time was both adopted and endorsed by the two most important philosophical writers about time in the early to mid-twentieth century, C. D. Broad and D. C. Williams (Savitt, 2002). This is the notion to which a relativistic notion of passage must be a counterpart or successor.

But we take it that another constraint on the relativistic successor concept to the commonsense concept of passage was set by Einstein (1949: 61):

We shall now inquire into the insights of definite nature which physics owes to the special theory of relativity.

(1) There is no such thing as simultaneity of distant events...

As we see it, what survives in special relativity is the notion of a succession of presents, but not a succession of presents built upon the notion of distant simultaneity. In our view, that leaves either a succession of events on a worldline or a succession of our local presents along a worldline as the best candidates for the relativistic counterpart of 
passage. It is not, we agree, easy to think of the passage of time as a local phenomenon, but we think that we have to learn to do so if the lessons of the special theory are to be fully appreciated.

Finally, the ideas and arguments of this chapter are not given solely for the purpose of assuaging certain sorts of anxieties, even those of Einstein. Our aim is to address certain sorts of deep intellectual perplexities, time being a deeply puzzling phenomenon. Our hope is that, insofar as any of these perplexities are mitigated by our ideas, the frustrating and so far intractable difficulties posed by time for the unification of relativity with quantum theory may come to be eased as well. ${ }^{28}$

\section{REFERENCES}

Arthur, Richard. 2008. Time lapse and the degeneracy of time: Gödel, proper time and becoming in relativity theory. In The ontology of spacetime II, ed. Dennis Dieks, 207-28. Amsterdam: Elsevier.

Arthur, Richard. 2010. Minkowski's proper time and the status of the clock hypothesis. In Space, time, and spacetime: Fundamental theories of physics, ed. Vesselin Petkov, 159-79. Berlin: Springer-Verlag.

Austin, John L. 1962. Sense and sensibilia. Oxford: Oxford University Press.

\footnotetext{
${ }^{28}$ Richard Arthur and Dennis Dieks have helped to make this essay much better than it otherwise would have been.
} 
Butterfield, Jeremy. 1984. Seeing the present. Mind 93: 161-76.

Callender, Craig. 2000. Shedding light on time. Philosophy of Science 67: S587-S599.

Callender, Craig. 2008. The common now. Philosophical Issues 18: 339-361.

Carnap, Rudolf. 1963. Intellectual autobiography. In The Philosophy of Rudolf Carnap, ed. Paul Arthur Schilpp, 3-84. LaSalle, Illinois: Open Court.

Clifton, Rob and Mark Hogarth. 1995. The definability of objective becoming in Minkowski spacetime. Synthese 103: 355-387.

Dorato, Mauro. 2008. Putnam on time and special relativity: A long journey from ontology to ethics. European journal of analytic philosophy 4: 51-70.

Einstein, Albert. 1949. Autobiographical notes. In Albert Einstein: philosopher-scientist, ed. Paul Arthur Schilpp, 2-94. La Salle, Illinois: Open Court.

Geroch, Robert. 1978. General relativity from A to B. Chicago: The University of Chicago Press.

Gödel, Kurt. 1949. A remark about the relationship between relativity theory and idealistic philosophy. In Albert Einstein: philosopher-scientist, ed. Paul Arthur Schilpp, 557-562. La Salle, Illinois: Open Court.

Halvorson, Hans. 2007. Algebraic quantum field theory. In Philosophy of Physics, Part $A$, eds. Jeremy Butterfield and John Earman, 731-864. Amsterdam: Elsevier.

Hestevold, H. Scott. 2008. Presentism: through thick and thin. Pacific Philosophical Quarterly 89: 325-47.

Maxwell, Nicholas. 1985. Are probabilism and special relativity incompatible? Philosophy of Science 52: 23-43.

Maxwell, Nicholas. 1988. Discussion: are Probabilism and special relativity incompatible? Philosophy of Science. 55: 640-645.

Myrvold, Wayne. 2003. Relativistic quantum becoming. British Journal for the Philosophy of Science 54: 475-500.

Penrose, Roger. 1972. Techniques of differential topology in relativity. Philadelphia: Society for Industrial and Applied Mathematics.

Penrose, Roger. 1989. The emperor's new mind. Oxford: Oxford University Press.

Putnam, Hilary. 1967. Time and physical geometry. The Journal of Philosophy 64: 240247. 
Putnam, Hilary. 2008. Reply to Mauro Dorato. European journal of analytic philosophy 4: 71-73.

Rietdijk, Cornelis Willem. 1966. A rigorous proof of determinism derived from the special theory of relativity. Philosophy of Science 33:341-344.

Rietdijk, Cornelis Willem. 1976. Discussion: special relativity and determinism. Philosophy of Science 43: 598-609.

Saunders, Simon. 2000. Tense and indeterminateness. Philosophy of Science 67: S600S611.

Savitt, Steven. 2002. On absolute becoming and the myth of passage. In Time, reality \& experience, ed. Craig Callender, 153-167. Cambridge: Cambridge University Press.

Savitt, Steven. 2006. Presentism and eternalism in perspective. In The ontology of spacetime, ed. Dennis Dieks, 111-27. Amsterdam: Elsevier.

Savitt, Steven. 2009. The transient nows. In Quantum reality, relativistic causality, and closing the epistemic circle, eds Wayne Myrvold and Joy Christian, 339-352. Springer.

Savitt, Steven. 2011. Time in the special theory of relativity. In The Oxford Handbook of Time, ed. Craig Callender. Oxford: Oxford University Press.

Sellars, Wilfrid. 1963. Philosophy and the scientific image of man. In Science, perception and reality, 1-40. New York: The Humanities Press

Stein, Howard. 1968. On Einstein-Minkowski space-time. The Journal of Philosophy 65: 5-23.

Stein, Howard. 1991. On relativity theory and the openness of the future. Philosophy of Science 58: 147-167.

Wüthrich, Christian. Forthcoming (in EPSA 2). Demarcating presentism. 